\title{
Evaluation of micronutrient levels in children and adolescents with obesity and their correlation with the components of metabolic syndrome
}

\author{
Fatih Kardaş ${ }^{1 \oplus}$, Arife Derda Yücel ${ }^{2 \oplus}$, Mustafa Kendirci ${ }^{3 \oplus}$, Selim Kurtoğlu ${ }^{3 \oplus}$, \\ Nihal Hatipoğlu ${ }^{3 \oplus}$, Leyla Akın ${ }^{3 \oplus}$, Ülkü Gül ${ }^{3 \oplus}$, Songül Gökay ${ }^{4 \oplus}$, \\ Pembe Soylu Üstkoyuncu ${ }^{4}$ \\ Divisions of ${ }^{1}$ Pediatric Nutrition and Metabolism and ${ }^{3}$ Pediatric Endocrinology, ${ }^{2}$ Department of Pediatrics, Erciyes University Faculty \\ of Medicine, ${ }^{4}$ Deparment of Pediatric Metabolism, Ministry of Health, Kayseri City Hospital, Kayseri, Turkey.
}

\begin{abstract}
Background. Obesity is a significant public health problem worldwide. Vitamin deficiencies, developing due to monotype nutrition, are more likely to be observed in patients than healthy children. The present study evaluates vitamin and micronutrient levels in children and adolescents with obesity and metabolic syndrome compared to healthy controls.
\end{abstract}

Methods. The study included 73 patients with obesity, 64 patients with metabolic syndrome and 71 healthy children (control group) aged 10 to 16 years. Physical examinations were performed, and waist circumference and systolic blood pressure measurements were recorded. Fasting blood glucose, triglyceride, high-density lipoprotein cholesterol, low-density lipoprotein cholesterol, total cholesterol, insulin, vitamin A, vitamin E, vitamin B1, vitamin B2, vitamin B6, vitamin B12, folic acid and free carnitine levels were analyzed. The homeostatic model of assessment-insulin resistance (HOMA-IR) index was calculated and recorded.

Results. The mean age of all patients was $11.9 \pm 2.6$ years. The serum insulin level and HOMA-IR index were found to be significantly higher in the obesity and metabolic syndrome groups. No significant difference was found between the groups in terms of vitamin A, vitamin B6 and free carnitine levels. Significantly decreased vitamin E, vitamin B2, vitamin B12 and folic acid and increased vitamin B1 levels were observed in the obesity and metabolic syndrome groups.

Conclusions. Compared to healthy children, children with obesity and metabolic syndrome may have varying degrees of micronutrient and vitamin deficiency due to poor and unbalanced eating habits. These deficiencies should also be considered in the treatment and follow-up of obesity and metabolic syndrome.

Key words: metabolic syndrome, micronutrient, obesity, vitamin.

Obesity is an energy metabolism disorder caused by excessive fat storage in the body, which may also cause physical and mental problems. Obesity is characterized by hypertension, impaired lipid profile and impaired glucose metabolism in addition to the development of insulin resistance, which forms the basis of the

$\bowtie$ Fatih Kardaş

fkardas@erciyes.edu.tr

Received 1st March 2020, revised 24th April 2020,

31st May 2020, accepted 9th June 2020. metabolic syndrome. ${ }^{1}$ The prevalence of obesity is increasing worldwide. Besides, metabolic syndrome constitutes a severe health problem in children and adolescents. ${ }^{2}$

It is assumed that, in exogenous obesity, micronutrient deficiencies can be seen not only due to carbohydrate and protein deficiency but also due to monotype or unbalanced nutrition. The present study hypothesizes that obesity and metabolic syndrome occur as a result of malnutrition and mainly monotype nutrition, and thus, a deficiency in various 
micronutrients. The World Health Organization (WHO) describes this situation as the "double burden of malnutrition", a reference to the coexistence of poor nutrition and excess weight or obesity, and diet-related non-communicable diseases within individuals, households and populations, and over an individual's lifespan. ${ }^{3}$ In recent years, based on this idea, new studies have been conducted. The fact that foods, which are rich in calories but nutrient deficient, are cheap and that their accessibility has become easier contributes to the development of obesity. ${ }^{4-6}$ Many micronutrients containing vitamins $\mathrm{A}, \mathrm{C}, \mathrm{D}$ and $\mathrm{E}$ were consumed in insufficient quantities by most obese adults and at significantly low levels in terms of total diet quality, fruit and micronutrient intake compared to healthy adults.?

In a study on obese children, the vitamin A level in the obesity group was significantly lower than that of the healthy control group. ${ }^{8}$ In different studies, alpha tocopherol and beta carotene levels were found to be lower in obese children compared to those in the control groups. ${ }^{9-11}$ In adults with metabolic syndrome, vitamin B1 levels were found to be lower than those in the control group. ${ }^{12}$ An inverse correlation has been reported between thiamine and vitamin B2 levels and body mass index (BMI) in obese children. ${ }^{13}$ The vitamin B6 level was found to be lower in patients with metabolic syndrome than that in the healthy population. ${ }^{12}$ Vitamin B12 deficiency was higher in obese patients compared to patients with a normal BMI. ${ }^{14}$ It has been shown in human and animal studies that carnitine supplementation improves glucose tolerance, especially in insulin-resistant patients. ${ }^{15}$ Studies on micronutrients in the obese population have generally been done in adults. A limited number of micronutrient levels were evaluated in a few studies in obese children.

Vitamin A, vitamin E, vitamin B1 (thiamine), vitamin B2 (riboflavin), vitamin B6 (pyridoxine), vitamin B12 (cobalamin), folic acid and free carnitine levels were included in the scope of the study, and it is difficult to comment on them individually in the first stage. The purpose of our research is to evaluate which micronutrient levels are affected in obese children and adolescents or in those with metabolic syndrome and determine their correlation with the components of metabolic syndrome.

\section{Material and Methods}

\section{Patients}

A written and verbal informed consent was obtained from the parents of all children. The study protocol was approved by the Ethics Committee of Erciyes University, Medical Faculty on 09.01.2015 with the report number 2015/23.

Among patients who were admitted to the Pediatric Metabolism Clinic of the Children's Hospital at Erciyes University Faculty of Medicine (Kayseri Province, Turkey) between April 2016 and April 2018, patients aged 10 to 16 years with a BMI Z-score $>2$ were included in the obesity group. ${ }^{16,17}$ Most of the children were from Central Turkey.

The patients aged 10 to 16 years meeting at least two international diabetes federation (IDF) criteria (triglyceride level $\geq 150 \mathrm{mg} / \mathrm{dl}$, high-density lipoprotein cholesterol (HDL-C) level $\leq 40 \mathrm{mg} / \mathrm{dl}$, systolic blood pressure $\geq 130$ $\mathrm{mmHg}$ or diastolic blood pressure $\geq 85 \mathrm{mmHg}$, and fasting plasma glucose level $\geq 100 \mathrm{mg} / \mathrm{dl}$ ) in addition to central obesity (waist circumference: $\geq 90 p$ ) were included in the metabolic syndrome group. ${ }^{18}$

The control group consisted of children aged 10 to 16 years, who had no known chronic disease and whose weight and height were within normal limits.

Height and weight measurements were performed with standard devices. BMI was calculated as weight (in kilograms) divided by height (in meters) squared. Waist circumference (WC) was measured at the end of expiration at the level of maximum waist narrowing. They 
were evaluated based on normative WC values in Turkish children. ${ }^{14}$ Children with a BMI at or above the $95^{\text {th }}$ percentile were classified as obese using Turkish children growth charts based on age and gender. ${ }^{11}$ BMI Z-scores were calculated using a software program "WHO AnthroPlusv1.0.4", and following cutoffs for BMI Z-scores defined by the WHO were used: obesity; BMI Z-score > 2, normal-weight; $-2<$ BMI Z-score $\leq 1 .{ }^{17}$

\section{Biochemical Parameters}

The blood samples for laboratory tests were taken following a 12-hour fasting period. Fasting blood glucose, triglyceride, HDL-C, low-density lipoprotein cholesterol (LDL-C), total cholesterol (T. Cholesterol), insulin, folic acid and vitamin B12 levels were analyzed and recorded. The biochemical parameters were analyzed using the Cobas 8000 C702 device (Germany) and standard assay kits (Roche, Germany) based on a spectrophotometric method. Insulin, vitamin B12 and folic acid levels were analyzed using the electrochemiluminescence method using Cobas8000 E602 (Germany) device and Roche (Germany) kits.

Serum, plasma and whole blood samples were stored at $-80{ }^{\circ} \mathrm{C}$ until the analysis of vitamin A, vitamin E, vitamin B1, vitamin B2, vitamin B6 and free carnitine levels were carried out. Vitamin A and E levels were analyzed using the high-pressure liquid chromatography (HPLC) method with a Thermo HPLC Spectra System (USA) device and Zivak (Turkey) kits. Vitamin B1, vitamin B2, vitamin B6, carnitine levels were analyzed using HPLC methods with an Agilent 1100 series HPLC device (Germany) and Recipe (Germany) kits.

HOMA-IR= Fasting Insulin $(\mu \mathrm{U} / \mathrm{mL}) \times$ Fasting Blood Glucose ${ }^{*}(\mathrm{mmol} / \mathrm{L}) / 22.5$

*Blood Glucose was calculated using mg/ $\mathrm{dl} / 18=\mathrm{mmol} / \mathrm{L}$ formula.

\section{Statistical Analysis}

The data were analyzed using the SPSS (Statistical Package for Social Sciences) for
Windows 22.0. Descriptive statistics were presented as mean \pm standard deviation or median (minimum-maximum) for continuous variables. Nominal variables were presented as several cases and percentages. The percentages between the groups were compared using the Chi-square test. In the comparison of the mean values between the two groups, the t-test was used if the data were normally distributed, and the Mann-Whitney test was used if the data were not normally distributed. Nonparametric data were analyzed using the Chi-square, Kruskal -Wallis or Mann Whitney U tests. The data with normal distribution were analyzed using the Pearson (r) correlation coefficient, while those without normal distribution were analyzed using Kendall's tau correlation coefficient. Also, multiple linear regression analysis, which is a more advanced statistical method, was used to evaluate the correlation between micronutrient values and the parameters of metabolic syndrome obtained in each group [ $[$ regression coefficient was used. $\mathrm{R}^{2}$ was considered as the coefficient of determination. It was interpreted that the closer the $R^{2}$ was to 1 , the greater part of the change in the dependent variable could be explained by the independent variable(s)]. For each micronutrient, the forward insertion method was used to identify the most significant clinical markers. The results were evaluated at a significance level of $\mathrm{p}<0.05$.

\section{Results}

\section{General characteristics of the study population}

The clinical characteristics of all patients are presented in Table I.

\section{Biochemical parameters and micronutrient levels}

Comparisons of biochemical and micronutrient levels between the groups are shown in Table II. Serum triglycerides were higher in the metabolic syndrome group than in the obesity and control groups ( $\mathrm{p}=0.001, \mathrm{p}=0.00$, respectively). 
Table I. Age, gender and anthropometric characteristics of all groups.

\begin{tabular}{lcccc}
\hline Parameters & $\begin{array}{c}\text { Obesity group } \\
(\mathrm{n}=73)\end{array}$ & $\begin{array}{c}\text { Metabolic syndrome group } \\
(\mathrm{n}=64)\end{array}$ & $\begin{array}{c}\text { Control group } \\
(\mathrm{n}=71)\end{array}$ & $\mathrm{p}$ \\
\hline Age (in years) & $12.5 \pm 2.3$ & $12.4 \pm 2.5$ & $11.2 \pm 1.8$ & 0.061 \\
Gender & & & & \\
$\quad$ Female & 36 & 32 & 37 & 0.885 \\
$\quad$ Male & 37 & 32 & 34 & 0.451 \\
Body weight $(\mathrm{kg})$ & $68.7 \pm 17.9^{\mathrm{a}}$ & $72.8 \pm 23.4^{\mathrm{b}}$ & $36.6 \pm 10.2^{\mathrm{a}, \mathrm{b}}$ & 0.001 \\
Height $(\mathrm{cm})$ & $152.2 \pm 13.3$ & $155.4 \pm 13.8$ & $142 \pm 11.7$ & 0.052 \\
WC $(\mathrm{cm})$ & $84.5 \pm 9.7^{\mathrm{a}}$ & $91.2 \pm 3^{\mathrm{b}}$ & $64.3 \pm 5.1^{\mathrm{a}, \mathrm{b}}$ & 0.001 \\
BMI $\left(\mathrm{kg} / \mathrm{m}^{2}\right)$ & $29 \pm 4.1^{\mathrm{a}}$ & $30.5 \pm 4.2^{\mathrm{b}}$ & $17.8 \pm 2.7^{\mathrm{a}, \mathrm{b}}$ & 0.001 \\
BMI Z-score & $2.46 \pm 0.41^{\mathrm{a}}$ & $2.54 \pm 0.47^{\mathrm{b}}$ & $-0.06 \pm 0.79^{\mathrm{a}, \mathrm{b}}$ & 0.001 \\
\hline
\end{tabular}

Data were expressed as the mean \pm SD.

a. Difference between obesity and control groups as: $\mathrm{p}<0.05$

b. Difference between metabolic syndrome and control groups as: $\mathrm{p}<0.05$

WC: Waist circumference, BMI: Body mass index.

Table II. Comparison of biochemical and micronutrient levels between groups.

\begin{tabular}{lcccc}
\hline Parameters & $\begin{array}{c}\text { Obesity group } \\
(\mathrm{n}=73)\end{array}$ & $\begin{array}{c}\text { Metabolic syndrome group } \\
(\mathrm{n}=64)\end{array}$ & $\begin{array}{c}\text { Control group } \\
(\mathrm{n}=71)\end{array}$ & $\mathrm{p}$ \\
\hline Triglycerides $(\mathrm{mg} / \mathrm{dl})$ & $82.8 \pm 29.3^{\mathrm{a}}$ & $138.7 \pm 73.2^{\mathrm{a}, \mathrm{c}}$ & $92.3 \pm 39.2^{\mathrm{c}}$ & 0.001 \\
HDL-C $(\mathrm{mg} / \mathrm{dl})$ & $49.4 \pm 6.3^{\mathrm{a}}$ & $41.8 \pm 10.6^{\mathrm{a}, \mathrm{c}}$ & $49.8 \pm 14.3^{\mathrm{c}}$ & 0.001 \\
T.Cholesterol $(\mathrm{mg} / \mathrm{dl})$ & $152.6 \pm 25.4$ & $168.4 \pm 61.3$ & $144.6 \pm 30.6$ & 0.063 \\
LDL-C $(\mathrm{mg} / \mathrm{dl})$ & $87.4 \pm 23.6$ & $96.7 \pm 56.5^{\mathrm{c}}$ & $77.4 \pm 29.9^{\mathrm{c}}$ & 0.033 \\
Fasting Glucose $(\mathrm{mg} / \mathrm{dl})$ & $88.6 \pm 7.8$ & $88.4 \pm 11$ & $87.8 \pm 11.1$ & 0.054 \\
Insulin $(\mu \mathrm{U} / \mathrm{mL})$ & $15.4 \pm 7^{\mathrm{b}}$ & $17.6 \pm 11.5^{\mathrm{c}}$ & $12 \pm 10.1^{\mathrm{b}, \mathrm{c}}$ & 0.001 \\
HOMA-IR & $3.3 \pm 1.7^{\mathrm{b}}$ & $3.7 \pm 2.4^{\mathrm{c}}$ & $2.4 \pm 2.3^{\mathrm{b}, \mathrm{c}}$ & 0.001 \\
Vitamin A $(0.3-0.7 \mathrm{mg} / \mathrm{L})$ & $0.7 \pm 0.4$ & $0.8 \pm 0.4$ & $0.8 \pm 0.3$ & 0.790 \\
Vitamin E $(5-20 \mathrm{mg} / \mathrm{L})$ & $11.5 \pm 12.2^{\mathrm{a}, \mathrm{b}}$ & $15.6 \pm 12.2^{\mathrm{a}, \mathrm{c}}$ & $29.9 \pm 10.7^{\mathrm{b}, \mathrm{c}}$ & 0.001 \\
Vitamin B1 $(25-85 \mu \mathrm{g} / \mathrm{L})$ & $46 \pm 17.3^{\mathrm{b}}$ & $45.8 \pm 22^{\mathrm{c}}$ & $36 \pm 12.9^{\mathrm{b}, \mathrm{c}}$ & 0.011 \\
Vitamin B2 $(40-240 \mu \mathrm{g} / \mathrm{L})$ & $89.1 \pm 35^{\mathrm{b}}$ & $116.7 \pm 65.2^{\mathrm{c}}$ & $174.5 \pm 51^{\mathrm{b}, \mathrm{c}}$ & 0.001 \\
Vitamin B6 $(3.6-18 \mu \mathrm{g} / \mathrm{L})$ & $11.9 \pm 4.8$ & $11.9 \pm 5.5$ & $13.6 \pm 7.8$ & 0.200 \\
Vitamin B12 $(197-771 \mathrm{pg} / \mathrm{ml})$ & $311.8 \pm 134.8^{\mathrm{b}}$ & $317.7 \pm 138.1^{\mathrm{c}}$ & $430.7 \pm 129.6^{\mathrm{b}, \mathrm{c}}$ & 0.002 \\
Folic acid $(3.9-26.8 \mathrm{ng} / \mathrm{ml})$ & $11 \pm 2.9^{\mathrm{b}}$ & $10.4 \pm 2.8^{\mathrm{c}}$ & $12.9 \pm 3.8^{\mathrm{b}, \mathrm{c}}$ & 0.012 \\
Free carnitine $(8.6-85 \mu \mathrm{mol} / \mathrm{L})$ & $27.1 \pm 14.8$ & $23.8 \pm 11.7$ & $21.4 \pm 11.5$ & 0.225 \\
\hline
\end{tabular}

Data were expressed as the mean \pm SD.

a. Difference between obesity and metabolic syndrome groups as: $p<0.05$

b. Difference between obesity and control groups as: $p<0.05$

c. Difference between metabolic syndrome and control groups as: $p<0.05$

HDL-C: high-density lipoprotein cholesterol, LDL-C: low-density lipoprotein cholesterol, T.Cholesterol: total cholesterol,

HOMA-IR: homeostatic model of assessment-insulin resistance.

HDL-C levels were found to be significantly lower in the metabolic syndrome group than in the obesity and control groups $(\mathrm{p}=0.001, \mathrm{p}=$ 0.001 , respectively). These two parameters are also metabolic syndrome criteria.
LDL-C levels were higher in the metabolic syndrome group than the control group $(p=0.014)$. Both of the obesity and metabolic syndrome groups' insulin and HOMA-IR levels were higher than the control group $(\mathrm{p}=0.001, \mathrm{p}=$ $0.001, p=0.001, p=0.001$ respectively). 
The vitamin E levels were found to be higher in patients with metabolic syndrome than those in obese children $(p=0.007)$.

Higher vitamin B1 levels were observed in the obesity and metabolic syndrome group than those in the control group $(\mathrm{p}=0.06, \mathrm{p}=0.020$ respectively).

The vitamin B2 values were lower in the obesity group and the metabolic syndrome group than in the control group $(\mathrm{p}=0.001, \mathrm{p}=0.001$ respectively).

The vitamin B12 level was lower in the obesity group compared to that in the control group $(p=0.001)$. A significant decrease was found in the vitamin B12 level in the metabolic syndrome group compared to that in the control group $(\mathrm{p}=$ $0.011)$.

Folic acid levels were higher in the control group compared to those in the obesity and the metabolic syndrome groups $(p=0.030, p=0.006$ respectively).

Correlation of micronutrient levels with, total cholesterol, LDL, HDL, HOMA-IR, fasting blood glucose, body weight, WC, and BMI $\mathrm{Z}$-score values in groups are indicated in Tables III, IV, and V.

In the obesity group; the vitamin B12 level was affected negatively by body weight and BMI Z-score $(B=-0.647, p=0.003, \beta=-0.249, p=0.017$ respectively).

In the metabolic syndrome group; the vitamin B2 level was independently affected negatively by triglyceride, HDL-C (two of the metabolic syndrome criteria) and LDL-C, and positively by total cholesterol. $(B=-1.667, p=0.002 ; \beta=-$ 5.713, $p=0.005, \beta=-0.968, p=0.013, \beta=-6.211$, $\mathrm{p}=0.005$ respectively) (Fig. 1 ).

The vitamin B12 level was correlated negatively with body weight $(\Omega=-0.281, \mathrm{p}=0.046)$.

In the control group; It was found that the vitamin B12 level was affected negatively by body weight in the control group $(B=-0.322, p=$ $0.014)$.

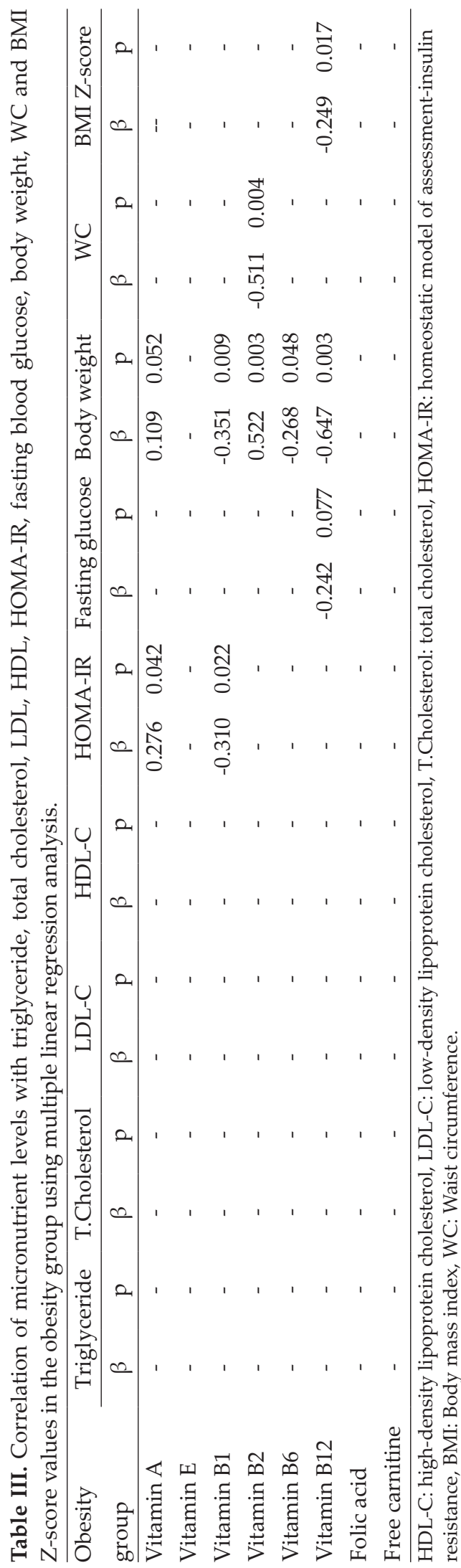

The Turkish Journal of Pediatrics • January-February 2021 

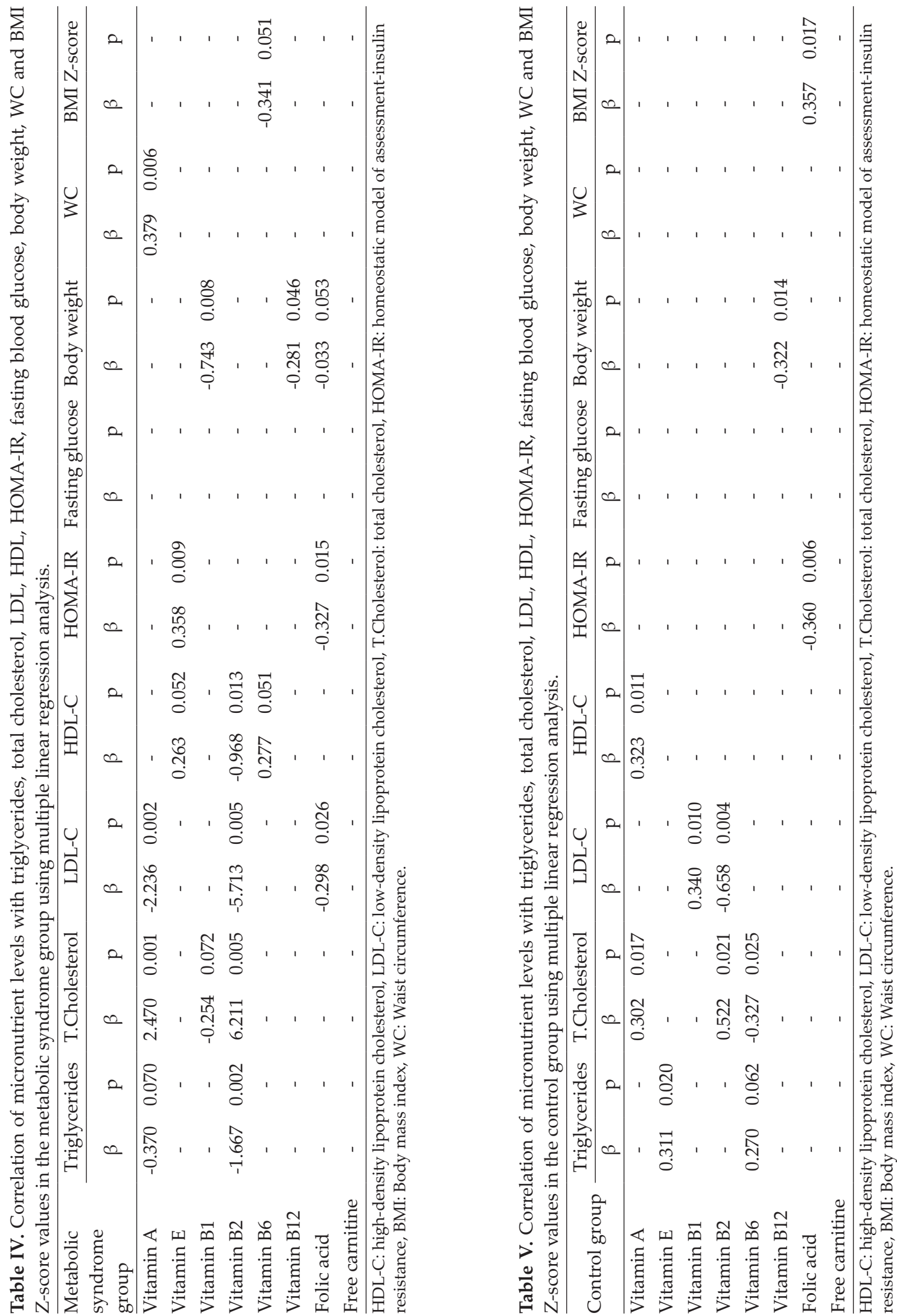


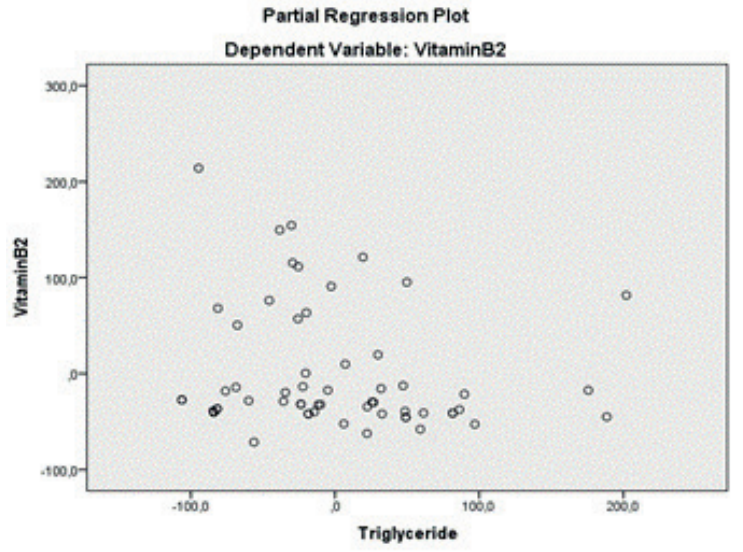

Fig. 1. Correlation graphics by multilinear regression analysis of vitamin B2 and triglyceride levels in the metabolic syndrome group.

Triglyceride affects vitamin B2 levels statistically significantly and negatively. A one unit increase in the triglyceride level decreases the vitamin B2 level by 1.667 units.

The vitamin B12 level was affected negatively by BMI Z-score among total subjects $(B=-0.254$, $\mathrm{p}=0.004$ ) (Fig. 2), but the vitamin B1 level was positively correlated by BMI Z-score for total children ( $\$=0.250, p=0.005)$ (Fig. 3).

In the obesity group, acanthosis was observed in five children and stria was observed in 30 patients. Also, in the metabolic syndrome group, 23 children with acanthosis and 36 children with

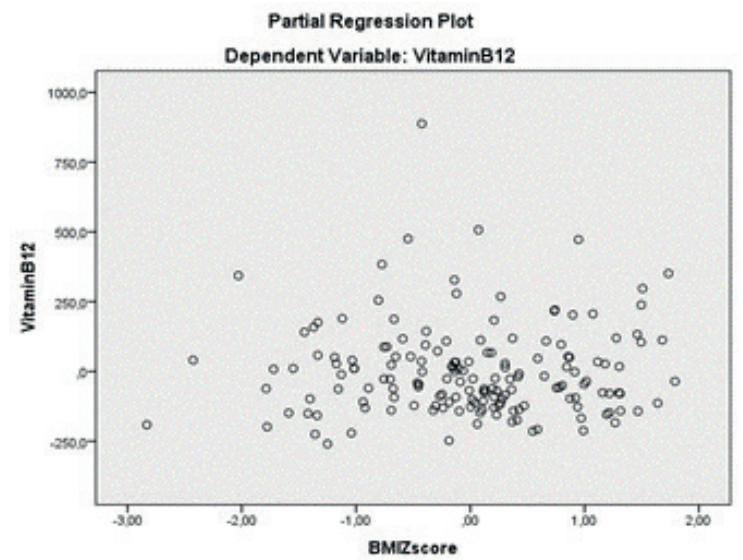

Fig. 2. Correlation graphics by multilinear regression analysis of vitamin B12 and BMI Z-scores in all groups.

BMI Z-score affects vitamin B12 levels statistically significantly and negatively. A one unit increase in the BMI Z-score decreases the vitamin B12 level by 0.254 units. stria were identified. The presence of acanthosis or stria was not associated with the levels of micronutrients or biochemical parameters for all groups or each group individually.

\section{Discussion}

In this study, micronutrient levels of children with obesity and metabolic syndrome were compared with each other and the control group. Thus, the relationship between micronutrients with metabolic syndrome criteria and their effects on obesity was analyzed.

According to us, obesity is a kind of malnutrition, which develops unilaterally and as a result of unbalanced nutrition. Hence, certain micronutrient deficiencies may develop in children who have an unbalanced diet. The WHO describes this situation as the "double burden of malnutrition" and points out that obesity may occur more frequently in socioeconomically underdeveloped and poorly nourished societies together with malnutrition, and that chronic diseases may develop as a consequence of this situation. ${ }^{3}$ In recent years, overweight cases have become more prevalent than underweight cases in underdeveloped and moderately developed countries. ${ }^{19}$

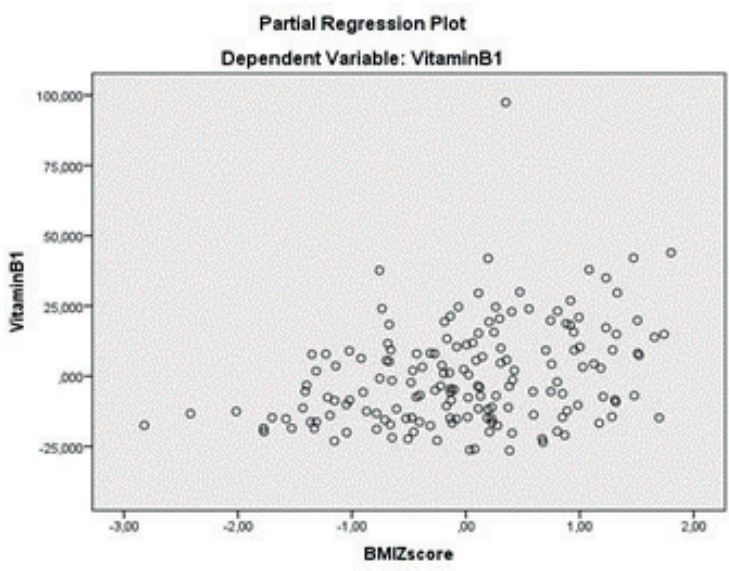

Fig. 3. Correlation graphics by multilinear regression analysis of vitamin B1 and BMI Z-scores in all groups.

BMI Z-score affects vitamin B1 levels statistically significantly and positively. A one unit increase in the BMI Z-score increases the vitamin B1 level by 0.250 units. 
In a study conducted in 2001-2008 on a nationally representative level of normalweight, overweight and obese adults, it was revealed that most micronutrients containing vitamins $\mathrm{A}, \mathrm{C}, \mathrm{D}$, and $\mathrm{E}$ as well as calcium and magnesium were deficient in most of the subjects in the study. This study also emphasized that obese individuals were significantly at low levels in terms of fruit intake, total diet quality and micronutrient intake compared to normals. ${ }^{7}$ Apart from the fact that obese patients may consume fewer micronutrients due to the foods they eat, micronutrient deficiencies may result from altered pharmacokinetics involving distribution, metabolism and elimination in obese patients as well. ${ }^{20}$ It is known that the pharmacokinetics of drugs are altered due to reasons such as increased adipose tissue, altered blood stream, increase or growth of liver parenchymal cells, and alteration of renal functions in obese people. ${ }^{21}$ Micronutrients may also be affected by similar mechanisms.

Previous studies on obese children show that vitamin A levels were significantly lower in obesity groups when compared to levels in healthy control groups, and that the vitamin A levels were directly proportional to BMI and abdominal obesity. ${ }^{22,23}$ However, in our study, no difference was found among the groups in terms of vitamin A levels (Table II).

Although recent studies suggest that beta carotene concentrations correlate inversely with HOMA-IR index, the present study showed a direct correlation between the HOMA-IR index and vitamin A level in the obesity group. ${ }^{24,25}$ In contrast to this finding, there was no significant correlation between the HOMA-IR index and vitamin A level in the metabolic syndrome group and the control group.

A recent study in overweight and obese patients found that vitamin A positively correlated with HLD-C and negatively correlated with LDL-C and triglycerides. But that study did not include metabolic syndrome. ${ }^{26}$ In our study, vitamin A levels positively correlated with LDL-C and total cholesterol in both metabolic syndrome and control groups while it negatively correlated with HDL-C levels in the metabolic syndrome group. The results of a previous study have shown that alphatocopherol levels are low in children with metabolic syndrome and at an average level in obese children, and it was speculated that obese children and children with metabolic syndrome are more prone to oxidative stress..$^{27}$ In the comparison of the three groups in our study, vitamin E levels were significantly lower in obese patients and those with metabolic syndrome. The children diagnosed with metabolic syndrome were given a Mediterranean diet, which is rich in fruits and vegetables. It was observed that metabolic syndrome criteria started to improve and vitamin E levels started to increase. ${ }^{28}$ Regarding the correlation between vitamin E levels and the parameters of metabolic syndrome, there was a positive correlation with the triglyceride levels in the control group and a statistically insignificant positive correlation with HDL-C in the metabolic syndrome group.

In the control group, there was a positive correlation between the vitamin B1 level and LDL-C. Vitamin B1 levels in adults were found to be lower in the metabolic syndrome group compared to the levels in the control group. Thiamine deficiency was found in children with obesity, and most of those cases were subclinical. ${ }^{12,29}$ In contrast to other studies, significantly higher vitamin B1 levels were found in the obesity group and those in the metabolic syndrome group compared to the levels in the control group. Interestingly, vitamin B1 levels were negatively correlated with the HOMA-IR and body weight in the obesity group, and with body weight in the metabolic syndrome group.

The reason for this difference may be explained by the fact that obese children and those with metabolic syndrome typically consume large quantities of cereal products, bread and potatoes, as cereals and potatoes are the main sources of vitamin B1. The association of increased thiamine intake and obesity has been revealed in other reports. ${ }^{30-32}$ 
An inverse correlation was found between BMI and vitamin B2 levels among obese children, and lower riboflavin levels were found in patients with metabolic syndrome. ${ }^{13,33}$ Our study revealed a negative correlation between B2 levels and body weight in the obesity group.

Examination of vitamin B2 levels in our study revealed that these levels were significantly lower in the obesity and metabolic syndrome groups than in the control group. Studies show that riboflavin deficiencies cause functional alterations in adipocytes, and thus have proinflammatory and proinsulin effects, which result from increased adipocytes. ${ }^{34}$

Although no correlation was found between vitamin B2 level and metabolic syndrome criteria in the obesity group, we found a negative correlation with LDL-C in the control group and a negative correlation with triglyceride, LDL-C, and HDL-C and a positive correlation with total cholesterol in the metabolic syndrome group.

It was noted that vitamin B6 supplementation is vital in preventing metabolic syndrome, and pyridoxine levels were lower in patients with metabolic syndrome compared to those in healthy patients. ${ }^{12,35}$ Unlike other studies, the vitamin B6 level in this study was normal, and there was no differences among the groups. In the obesity group, an inverse correlation was found between vitamin B6 and body weight. In the literature, there are not many studies that analyze the correlation between vitamin $\mathrm{B} 6$ and obesity in children.

In a few studies where obese children are compared with healthy children, vitamin B12 levels were found to be lower in obese children. ${ }^{14,36-38}$ In parallel to these findings, our results showed significant lower levels of B12 in the obesity and metabolic syndrome groups than the control group, and B12 levels were negatively correlated with body weight in each group. Also, the vitamin B12 was affected negatively by BMI Z-score among total subjects and in the obesity group.
It is suggested that there is an inverse correlation between folic acid levels and BMI, and that folic acid deficiency can play a role in the etiology of obesity. ${ }^{13}$ Our study revealed lower folic acid levels in children with obesity and metabolic syndrome compared to the control group. A lower intake of folic acid was observed in obese children compared to healthy children. ${ }^{39}$ It was shown that oral glucose tolerance induced insulin resistance in rats with diet-induced folic acid deficiency. ${ }^{15}$ Consistent with this study, we found a negative correlation between folic acid levels and HOMA-IR in the control and metabolic syndrome groups.

In human and animal studies, it has been shown that carnitine supplementation improves glucose tolerance, especially in insulin-resistant patients. However, more precise results can be obtained from animal studies investigating the correlation between carnitine deficiency and glucose intolerance. ${ }^{15}$ In our study, no significant difference was found among the groups in terms of the free carnitine level. Besides, there were no correlations in each group, between the biochemical parameters of metabolic syndrome and free carnitine levels in each group. A current meta-analysis shows a significant effect of L-carnitine supplementation as a potential therapeutic agent on weight and BMI in obese and overweight adults. ${ }^{40}$

The weaknesses of our study are that the socioeconomic and cultural status of families cannot be evaluated. Likewise, the average daily calorie and vitamin consumption levels of patients cannot be calculated using weekly food lists due to the families' lack of interest.

Considering that studies in the literature on this subject have been performed solely on adults to date, our study will be the first to obtain data findings on this subject, that will involve children with obesity and with metabolic syndrome in Turkey.

In conclusion, none of the micronutrients were measured below the normal range indicating a deficiency. In our study, the 
micronutrient content of the diet could not be calculated, because there was no nutritional record. However, measured vitamin levels can indirectly inform us about the vitamin intake of children based on their diets. The fact that higher vitamin B1 levels were found in the children with obesity and metabolic syndrome than healthy children in the control group suggests that these children consume more carbohydrates, which contains grain products, particularly cereal products (pastry etc.). The fact that lower vitamins E, B2, B12 and folic acid levels were found in children with obesity and metabolic syndrome than healthy children in the control group implies that these children do not consume as many green-leaved vegetables, dairies, specialty meats, seafood, citrus fruit and other animal products.

Primary healthcare institutions should ensure to be sensitive towards nutrition training and psychological counseling. The development of health policies for the prevention of obesity should be continued persistently.

\section{Acknowledgment}

This study was funded by the Scientific and Technological Research Council of Turkey. TÜBİTAK project number: $115 S 877$

\section{REFERENCES}

1. Rosende A, Pellegrini C, Iglesias R. Obesity and metabolic syndrome in children and adolescents. Medicina (B Aires) 2013; 73: 470-481.

2. Poyrazoğlu Ş, Baş F, Darendeliler F. Metabolic syndrome in young people. Curr Opin Endocrinol Diabetes Obes 2014; 21: 56-63.

3. World Health Organization. Nutrition. Double burden of malnutrition. 2020. from http://www. who.int/nutrition/double-burden-malnutrition/en/. (Retrieved at April 10,2020)

4. Swinburn BA, Sacks G, Hall KD, et al. The global obesity pandemic: shaped by global drivers and local environments. Lancet 2011; 378: 804-814.

5. Cutler DM, Glaeser EL, Shapiro JM. Why have Americans become more obese? J Econ Perspect 2003; 17: 93-118.
6. Via M. The malnutrition of obesity: micronutrient deficiencies that promote diabetes. ISRN Endocrinol 2012; 2012:103472.

7. Agarwal S, Reider C, Brooks JR, Fulgoni VL 3rd. Comparison of prevalence of inadequate nutrient intake based on body weight status of adults in the United States: an analysis of NHANES 2001-2008. J Am Coll Nutr 2015; 34: 126-34

8. Wei X, Peng R, Cao J, et al. Serum vitamin A status is associated with obesity and the metabolic syndrome among school-age children in Chongqing, China. Asia Pac J Clin Nutr 2016; 25: 563-570.

9. Decsi T, Molnar D, Koletzko B. Reduced plasma concentrations of alpha-tocopherol and betacarotene in obese boys. J Pediatr 1997; 130: 653-655.

10. Saccardo Sarni RO, Suano de Souza FI, Ramalho RA, et al. Serum retinol and total carotene concentrations in obese pre-school children. Med Sci Monit 2005; 11 : CR510-CR514.

11. Burrows TL, Warren JM, Colyvas K, Garg ML, Collins CE. Validation of overweight children's fruit and vegetable intake using plasma carotenoids. Obesity (Silver Spring) 2008; 17: 162-168.

12. Odum EP, Wakwe VC. Plasma concentrations of water-soluble vitamins in metabolic syndrome subjects. Niger J Clin Pract 2012; 15: 442-447.

13. Gunanti IR, Marks GC, Al-Mamun, A, Long KZ. Low serum vitamin B-12 and folate concentrations and low thiamin and riboflavin intakes are inversely associated with greater adiposity in Mexican American children. J Nutr 2014; 144: 2027-2033.

14. MacFarlane AJ, Greene-Finestone LS, Shi Y. Vitamin B-12 and homocysteine status in a folate-replete population: results from the Canadian Health Measures Survey. Am J Clin Nutr 2011; 94: 10791087.

15. Ringseis R, Keller J, Eder K. Role of carnitine in the regulation of glucose homeostasis and insulin sensitivity: evidence from in vivo and in vitro studies with carnitine supplementation and carnitine deficiency. Eur J Nutr 2012; 51: 1-18.

16. Neyzi O, Bundak R, Gokcay G, et al. Reference values for weight, height, head circumference, and body mass index in Turkish children. J Clin Res Pediatr Endocrinol 2015; 7: 280-293.

17. de Onis M, Onyango AW, Borghi E, Siyam A, Nishida C, Siekmann J. Development of WHO growht reference for school-aged children and adolescents. Bull World Health Organ 2007; 85: 660-667.

18. Zimmet P, Alberti KG, Kaufman F, et al. The metabolic syndrome in children and adolescents-an IDF concensus report. Pediatr Diabetes 2007; 8: 299306. 
19. Abdullah A. The double burden of undernutrition and overnutrition in developing countries: an Update. Curr Obes Rep 2015; 4: 337-349.

20. Zuckerman M, Greller HA, Babu KM.A Review of the Toxicologic implications of obesity. J Med Toxicol 2015; 11: 342-354.

21. Blouin RA, Kolpek JH, Mann HJ. Influence of obesity on drug disposition. Clin Pharm 1987; 6: 706-714.

22. Zwiauer KF. Prevention and treatment of overweight and obesity in children and adolescents. Eur J Pediatr 2000; 159(Suppl 1): 56-68

23. García OP, Ronquill, D, del Carmen Caamano M. Zinc, iron and vitamins $\mathrm{A}, \mathrm{C}$ and $\mathrm{E}$ are associated with obesity, inflammation, lipid profile and insulin resistance in Mexican school-aged children. Nutrients 2013; 5: 5012-5030.

24. Shin MJ, Park E. Contribution of insulin resistance to reduced antioxidant enzymes and vitamins in nonobese Korean children. Clin Chim Acta 2006; 365: 200-205.

25. Canas JA, Damaso L, Altomare A, Killen K, Hossain J, Balagopal PB. Insulin resistance and adiposity in relation to serum $\beta$-carotene levels. J Pediatr 2012; 161: 58-64.e2

26. Viroonudomphol D, Pongpaew P, Tungtrongchitr $\mathrm{R}$, et al. The relationships between anthropometric measurements, serum vitamin $A$ and $E$ concentrations and lipid profiles in overweight and obese subjects. Asia Pac J Clin Nutr 2003; 12: 73-79.

27. Molnar D, Decsi T, Koletzko BV. Reduced antioxidant status in obese children with multimetabolics yndrome. Int J Obes (Lond) 2004; 28: 1197-1202.

28. Velázquez-López L, Santiago-Díaz G, NavaHernández J, Muñoz-Torres AV, Medina-Bravo P, Torres-Tamayo M. Mediterranean-style diet reduces metabolic syndrome components in obese children and adolescents with obesity. BMC Pediatr 2014; 14: 175.

29. Densupsoontorn N, Srisawat C, Chotipanang K, et al. Prevalence of and factors associated with thiamin deficiency in obese Thai children. Asia Pac J Clin Nutr 2019; 28: 116-121.

30. Ortega RM, López-Sobaler AM, Andrés P, RodríguezRodríguez E, Aparicio A, Bermejo LM. Increasing consumption of breakfast cereal improves thiamine status in overweight/obese women following a hypocaloric diet. Int J Food Sci Nutr 2009; 60: 69-79.
31. Zhou SS, Zhou Y. Excess vitamin intake: an unrecognized risk factor for obesity. World J Diabetes 2014; 5: 1-13.

32. Zhou SS, Li D, Zhou YM, Sun WP, Liu QG. B-vitamin consumption and the prevalence of diabetes and obesity among the US adults: population based ecological study. BMC Public Health 2010; 10: 746.

33. Yu Y, Song Y. Three clustering patterns among metabolic syndrome risk factors and their associations with dietary factors in Korean adolescents: based on the Korea National Health and Nutrition Examination Survey of 2007-2010. Nutr Res Pract 2015; 9: 199-206.

34. Mazur-Bialy AI, Pocheć E.Vitamin B2 deficiency enhances the pro-inflammatory activity of adipocyte, consequences for insulin resistance and metabolic syndrome development. Life Sci 2017; 178: 9-16.

35. Liu Z, Li P, Zhao ZH, Zhang Y, Ma ZM, Wang SX. Vitamin B6 prevents endothelial dysfunction, insulin resistance, and hepatic lipid accumulation in apoe (-/-) mice fed with high-fat diet. J Diabetes Res 2016; 2016: 1748065.

36. Pinhas-Hamiel O, Doron-Panush N, Reichman B, Nitzan-Kaluski D, Shalitin S, Geva-Lerner L. Obese children and adolescents: a risk group for low vitamin B12 concentration. Arch Pediatr Adolesc Med 2006; 160: 933-936.

37. Narang M, Singh M, Dange, S. Serum homocysteine, vitamin B12 and folic acid levels in patients with metabolic syndrome. J Assoc Physicians India 2016; 64: 22-26.

38. Kozioł-Kozakowska A, Kozłowska M, Jagielski P. Assessment of diet quality, nutrient intake, and dietary behaviours in obese children compared to healthy children. Pediatr Endocrinol Diabetes Metab 2020; 26: 27-38.

39. Askarpour M, Hadi A, Miraghajani M, Symonds ME, Sheikhi A, Ghaedi E. Beneficial effects of 1-carnitine supplementation for weight management in overweight and obese adults: an updated systematic review and dose-response meta-analysis of randomized controlled trials. Pharmacol Res 2020; 151: 104554

40. Sun $Y$, Sun M, Liu B, et al. Inverse association between serum vitamin B12 concentration and obesity among adults in the united states. Front Endocrinol (Lausanne) 2019; 10: 414. 